\title{
Evaluation of Volatile Compounds of “Tiger Nut Beverage" (Orxata de Xufla) Headspace by Optimized Solid-Phase Micro-Extraction
}

\author{
Bruna Klein1, Joan-Josep Gallardo-Chacón², Idoia Codina-Torrella², Antonio-José Trujillo², \\ Bibiana Juan2* \\ ${ }^{1}$ Núcleo Integrado de Desenvolvimento em Análises Laboratoriais (NIDAL), Universidade Federal de Santa \\ Maria (UFSM), Santa Maria, Brasil \\ ${ }^{2}$ Centre Especial de Recerca Planta de Tecnologia dels Aliments (CERPTA), XaRTA, TECNIO, Departament de \\ Ciència Animal i dels Aliments, Universitat Autònoma de Barcelona, Barcelona, Spain \\ Email: ${ }^{*}$ bibiana.juan@uab.cat
}

Received 1 September 2014; revised 8 October 2014; accepted 9 November 2014

Copyright (C) 2014 by authors and OALib.

This work is licensed under the Creative Commons Attribution International License (CC BY).

http://creativecommons.org/licenses/by/4.0/

(c) (i) Open Access

\section{Abstract}

The aroma profile of "tiger nut beverage" (orxata de xufla) was investigated by headspace solidphase micro-extraction (HS-SPME) combined with gas chromatography-mass spectrometry (GCMS). Polidimethilsiloxane/divinylbenzene (PDMS/DVB), carboxen/polydimethylsiloxane (CAR/ PDMS), polyacrylate (PA) and divinylbenzene/carboxen/polydimethylsiloxane (DVB/CAR/PDMS) fibers were tested and compared for sensitivity beyond conditions that might affect the SPME procedure, such as temperature, concentration of ions and sample volume. The SPME fiber coated with DVB/CAR/PDMS presented the most efficient extraction compounds from the headspace of the product, particularly when the volatiles were extracted at $60^{\circ} \mathrm{C}$ with $2 \mathrm{~mL}$ of the sample volume. The addition of salt did not show any significant advantage for the qualification of the volatile fraction. Eighty-three compounds were identified or tentatively identified by GC-MS, being terpenes the most abundant group.

\section{Keywords}

Headspace Solid-Phase Micro-Extraction (SPME), Gas Chromatography-Mass Spectrometry (GC-MS), Tiger Nut Beverage, Volatile Compounds

Subject Areas: Analytical Chemistry, Food Science \& Technology

\footnotetext{
"Corresponding author.
}

How to cite this paper: Klein, B., Gallardo-Chacón, J.-J., Codina-Torrella, I., Trujillo, A.-J. and Juan, B. (2014) Evaluation of Volatile Compounds of "Tiger Nut Beverage" (Orxata de Xufla) Headspace by Optimized Solid-Phase Micro-Extraction. Open Access Library Journal, 1: e1015. http://dx.doi.org/10.4236/oalib.1101015 


\section{Introduction}

Nowadays, vegetal beverages are widely present in the European markets as a good alternative to cow milk. Their interest lies in lactose intolerant people or who is allergic to milk's proteins. Although these beverages are not nutritionally comparable with milk, they have been promoted as healthy foods, due to their properties and benefits against different human diseases [1].

Tiger nuts are nutritious tubers, which are widely cultivated across the Mediterranean areas as livestock food and for human consumption. In Spain, the economic importance of tiger nuts crop lies in the production of "orxata de xufla", a nutritious milky-like product mechanically obtained from the healthy, mature and selected tiger nuts. The tiger nut production in Spain was close 8 tonnes in 2010 [2], and according to an industrial production survey, 40 to 50 million liters of tiger nuts milk are manufactured every year, representing a retail market value of approximately 60 million euro [3].

The first step of tiger nut beverage production corresponds to the soaking of tubers. Then, soaked tiger nuts and water are ground, and the mixture is left to macerate, press and filter for obtaining the milky product. Finally, between 100 and $120 \mathrm{~g} / \mathrm{L}$ of sugar is added [4]. Tiger nut beverage is an energetic drink rich in starch and fat. It is also considered as a healthy and nourishing food; its profile is rich in oleic ( $75 \%$ of total fat) and linoleic acid ( $9 \%$ to $10 \%$ of total fat), and in addition to this, the essential amino acids in this beverage are higher than the amount in the model protein proposed for adults by the FAO/OMS [5]. Therefore, this product has a great potential in the food market, limited only by its very short shelf-life. Thermal processing is undoubtedly the most common method used to extend shelf-life by reducing microbial numbers and enzyme activity. Most commercial tiger nut beverages are subjected to a process technology that suppresses or transforms its starch content, and then they are treated with heat treatments (e.g., UHT) and packaged aseptically. Other kinds of tiger nut beverages are pasteurized tiger nut beverages, which have been subjected to thermal treatment below $72^{\circ} \mathrm{C}$ without addition of additives or processing aids. UHT tiger nut beverages presented a longer life than the pasteurized, and both were longer than traditional beverages, which were quickly sold after production and maintained under refrigeration conditions.

Aroma is a key determinant of quality and is a function of the volatile profile of a foodstuff. The chemical changes induced by processing and post-production alter the sensory profile of its specific components [6] and could initiate reactions that result in the formation of off-flavours [7], modifying the sensory perceptions of a freshly prepared product.

Solid-phase microextraction (SPME) has been reported to be relatively cheap, solventless, fast and reproducible, and it also eliminates problems associated with chemically and thermally instable samples [8]. SPME with gas chromatography-mass spectrometry (GC-MS), has been largely used for the determination of volatile compound profile in different foodstuff [9].

The objective of this work was the optimization of a method based on HS-SPME/CG-MS to describe qualitatively the volatile compounds of "tiger nut beverage". The parameters studied were four types of SPME fibers, coated with different stationary phases [PDMS/DBV, CAR/PDMS, DBV/CAR/PDMS and PA], used to examine their extraction efficiencies and compared for sensitivity beyond conditions that might affect the SPME procedure, such as temperature, concentration of ions and sample volume. This is the first report of volatiles in this food matrix.

\section{Materials and Methods}

\subsection{Samples}

Samples were purchased in local supermarkets. The experimental design for optimization of the analytical method was performed with samples of the tiger nut beverage of the same batch treated by ultra high temperature (UHT). Once the extraction conditions were established, eight UHT treated tiger nut beverage trademarks were analyzed. Also, a free additives commercial pasteurized sample was added to the study as a control. Table 1 describes the ingredients of the commercial tiger nut beverages analyzed in this study, in accordance with those specified on their labels. All solvent and analytical reagents were purchased by Sigma-Aldrich (St. Louis, MO, USA).

\subsection{Isolation of Volatile Compounds}

The volatile compounds of different commercial brands of tiger nut beverage were isolated using the technique 
Table 1. Ingredients present in the tiger nut beverages.

\begin{tabular}{|c|c|}
\hline Tiger nut milk & Ingredients \\
\hline OXT1 & $\begin{array}{l}\text { Water, tiger nuts, sugar, } \\
\text { emulsifier, milk proteins } \\
\text { antioxidant and flavourings }\end{array}$ \\
\hline OXT2 & $\begin{array}{l}\text { Water, tiger nuts, sugar, } \\
\text { emulsifier, milk proteins } \\
\text { antioxidant and flavourings }\end{array}$ \\
\hline ОХТ3 & $\begin{array}{l}\text { Water, tiger nuts, sugar, } \\
\text { emulsifier, milk proteins } \\
\text { antioxidant and flavourings }\end{array}$ \\
\hline OXT4 & $\begin{array}{l}\text { Water, tiger nuts, sugar, } \\
\text { emulsifier, milk proteins } \\
\text { and flavourings }\end{array}$ \\
\hline OXT5 & $\begin{array}{l}\text { Water, tiger nuts, sugar, } \\
\text { emulsifier, milk proteins } \\
\text { and flavourings }\end{array}$ \\
\hline OXT6 & $\begin{array}{l}\text { Water, tiger nuts, sugar, } \\
\text { emulsifier, milk proteins } \\
\text { antioxidant and flavourings }\end{array}$ \\
\hline OXT7 & $\begin{array}{l}\text { Water, tiger nuts, sugar, } \\
\text { emulsifier, milk proteins } \\
\text { and flavourings }\end{array}$ \\
\hline ОXT8 & $\begin{array}{l}\text { Water, tiger nuts, sugar cane, } \\
\text { emulsifier, stabilizer }\end{array}$ \\
\hline OXT9 & Water, tiger nuts, sugar \\
\hline
\end{tabular}

of solid-phase microextraction (SPME), where the fiber was exposed in the confined space (headspace) between the sample and the vial $(10 \mathrm{~mL})$ fitted with a silicone septum. A magnetic spinner was used to keep samples under stirring during the extraction (700 rpm).

\subsection{Selection of Fiber}

Four different SPME fibers were tested: PDMS/DVB (polydimethylsiloxane/divinylbenzene, supelco, $65 \mu \mathrm{m} \times$ $10 \mathrm{~mm}$ ), CAR/PDMS (carboxen/polydimethylsiloxane, supelco, $85 \mu \mathrm{m} \times 10 \mathrm{~mm}$ ) polyacrylate (PA, supelco, 85 $\mu \mathrm{m} \times 10 \mathrm{~mm}$ ) and DVB/CAR/PDMS (divinylbenzene/carboxen/polydimethylsiloxane, supelco, 50/30 $\mu \mathrm{m} \times 20$ $\mathrm{mm})$. Fibers were daily conditioned before use according to the manufacturer's instructions. In the preliminary selection, all the fibers were tested to select the one presenting the best capacity to extract "orxata" volatiles. In this step, all the fibers were exposed to the sample headspace under the following conditions: $4 \mathrm{~mL}$ of the sample, 10 min of equilibrium time, 60 min of extraction time and $40^{\circ} \mathrm{C}$ of extraction temperature (conditions arbitrarily established by the authors in the choice-of-fiber step). All the fibers were tested in triplicate.

\subsection{Multilevel Factorial Experimental Design}

Once the most suitable fiber for the analysis was chosen, it was proceeded to study the optimum conditions to establish extraction in headspace volatiles of tiger nut beverages. An experimental design was conducted with three variables at two levels that could affect the extraction of volatile compounds. The variables tested were the 
sample volume used in the extraction $(2 \mathrm{~mL}$ or $4 \mathrm{~mL})$, the extraction temperature $\left(40^{\circ} \mathrm{C}\right.$ or $\left.60^{\circ} \mathrm{C}\right)$ and the effect of increasing ionic strength of the medium by addition of $\mathrm{NaCl}$ in the sample until the saturation level (weight/volume). The extraction time of volatiles was set at $60 \mathrm{~min}$ by exposing the fiber in the headspace of the sample. In all experiments, the vial containing the sample plus $3 \mu \mathrm{L}$ internal standard (4-methyl-2-pentanol in methanol) was previously maintained for $10 \mathrm{~min}$ at the same temperature as the extraction (equilibrium time), and continuously homogenized by stirring with a magnetic stirrer at $700 \mathrm{rpm}$. All extractions were analyzed in triplicate. The choice of the parameters tested took into consideration the profile of volatile compounds, the number of peaks in the chromatogram, and especially the sum of the total area of the peaks.

\subsection{GC-MS Analysis}

Separation and identification of volatile compounds was performed using an Agilent 6890 gas chromatograph coupled to a 5975 MSD mass spectrometer (Agilent Technologies, Palo Alto, CA). A J\&W HP-INNOWAX capillary column $(60 \mathrm{~m} \times 0.251 \mathrm{~mm} \times 0.25 \mu \mathrm{m})$ with a bonded polyethylene glycol (PEG) high polarity stationary phase. The volatiles were desorbed in the injection port in splitless mode at $250^{\circ} \mathrm{C}$ for $3 \mathrm{~min}$, but the fiber was maintained in the injection port for a total of $15 \mathrm{~min}$ for cleaning. Helium was used as the carrier gas at a flow rate of $1 \mathrm{~mL} / \mathrm{min}(25.641 \mathrm{~cm} / \mathrm{s})$. Initial column temperature was kept at $40^{\circ} \mathrm{C}$ for $5 \mathrm{~min}$, and then increased to $220^{\circ} \mathrm{C}$ at $10^{\circ} \mathrm{C} / \mathrm{min}$, and held for $10 \mathrm{~min}$.

\subsection{Characterization of Volatiles in Tiger Nut Beverages}

Tentative identification of volatile compounds was achieved by comparing their mass spectra with those of mass spectra libraries [10]. Additionally, $\mathrm{C}_{8}$ through $\mathrm{C}_{20}$ aliphatic hydrocarbon standards dissolved in methanol (Sigma-Aldrich, St. Louis, MO, USA) and $\mathrm{C}_{9}$ through $\mathrm{C}_{36}$ in ethylene chloride (RESTEK, Ballefonte, PA, USA) were used to calculate linear retention indexes, which were compared with those reported in the literature. Available chemical standards were used to confirm volatiles identification. The results were expressed as micrograms of 4 methyl-2-pentanol/mL of tiger nut beverage.

Areas above the detection limit (LOD) determined by measuring the average noise and standard deviation value, was considered after analyzed 10 blanks (calibration matrix). Average noise plus 3 times standard deviation were used respectively for LOD.

\subsection{Statistical Analysis}

A multivariate analysis was applied using R statistical software [11] for the analysis of the experimental data for fiber selection. The significance of the factors studied in the experimental design and the optimum values for each factor (volume, $\mathrm{NaCl}$, temperature) were established by means analysis of variance (ANOVA), as well as multiple comparisons, using the Tukey's HSD test. The software Statistica v. 7 (Statsoft Inc., Tulsa, OK, USA) was used. Results were considered significant at $p$-value $<0.05$.

\section{Results and Discussion}

\subsection{Selection of Fiber}

An effective SPME method strongly depends on the fiber used. Volatile compounds were extracted with different fibers, tentatively identified by their mass spectrum and classified according to their functional groups. The sums of the mass spectrometer detector response (peak areas) for the volatile compounds identified were used to study the extraction efficiency of each fiber.

Fiber coatings often consist of two or three components, recovery capabilities of which are based on phenomena like absorption (PDMS) and adsorption (CAR/PDMS) [12]. Polyacrylate-coated fiber (PA) is used to extract very polar analytes from polar samples. DVB/PDMS, CAR/PDMS, and DVB/CAR/PDMS fibers are bipolar and extract compounds with different characteristics. The difference between CAR/PDMS and DVB/CAR/ PDMS fibers is that one of them contains divinyl benzene (DVB) while the other one does not. DVB is an aromatic non-polar phase that could be responsible for the extraction of a higher number of aromatics, alkanes, alkenes and terpenes [compounds with low molecular weight] via $\pi$-interactions. It is also suited for the analysis of semi-volatile compounds, and also serves, in conjunction with PDMS, as a sieve for bigger molecules in or- 
der to allow the smaller analytes to be adsorbed onto the CAR coating.

Table 2 shows the total number of compounds per functional group extracted per fiber and the sum of peak areas per functional groups, respectively. Among the four fibers used in this study, PA and PDMS/DVB did not have a satisfactory performance for the analysis of volatile profile of tiger nut beverage. In general, PA extracted fewer compounds and had the lowest sum of peak areas compared to other fibers. The fiber that extracted the greatest number of compounds was DVB/CAR/PDMS, followed by CAR/PDMS and PDMS/DVB. The response (assessed by peak area per functional group) of the fiber DVB/CAR/PDMS was significantly different to the CAR/PDMS for most functional groups evaluated. DVB/CAR/PDMS fiber had a greater sum of peak areas for aldehydes, aromatic compounds, esters, alkanes, alkenes and terpenes. Overall, the DVB/CAR/PDMS fiber gave a good extraction of volatile compounds, showing good sensitivity and selectivity for the extraction of a wide range of compounds with different polarities and molecular weights. The intermediate polarity of DVB/ CAR/PDMS, associated with the mixed nature of this coating (existence of meso-macropores resulting from the rugosity of the liquid PDMS film associated with the solid pores of CAR and DVB), would probably explain its capacity to extract a greater number of volatiles as compared to the others [13].

\subsection{Optimization of Extraction Parameters}

\section{Factorial Design}

To optimize the appropriate SPME extraction conditions we used the selected DVB/CAR/PDMS fiber and UHT-treated tiger nut beverage from one unique batch. The variables tested were salinity $(\mathrm{NaCl})$, extraction temperature (Temp), and sample volume (Vol) by way of the factorial design described. The optimal extraction conditions were statistically established by analyzing the factorial design results for the sixty-four variables (volatile compounds).

Table 3 shows that $\sim 68 \%$ of the volatile compounds were statistically affected by the extraction temperature factor $(p<0.05)$. Most of these variables were statistically better extracted at a temperature of $60^{\circ} \mathrm{C}$. At $40^{\circ} \mathrm{C}$, compounds with high volatilities were easily transported to the headspace, but compounds with lower volatilities required a higher temperature. Heating provides the energy for the volatile analytes to overcome the energy barriers which bind them to the matrix, enhancing the mass transfer process and increases vapor pressure of the volatiles, making easier their release to the headspace [14].

The sample volume had less effect on the overall extraction of compounds, only $~ 22 \%$ of compounds were statistically affected. However, for those variables that were statistically significant nearly all of them had a preference for the $2 \mathrm{~mL}$ volume. These results may be due to the higher responses of some compounds, such as

Table 2. Sum of peak areas $\left(\times 10^{5}\right)$ and (number of compounds) for the four fibers tested per functional group.

\begin{tabular}{ccccc}
\hline Functional group & DVB/CAR/PDMS & CAR/PDMS & PDMS/DVB & PA \\
\hline Aldehydes & $558.3^{\mathrm{a}}(10)$ & $343.7^{\mathrm{ab}}(8)$ & $271.7^{\mathrm{bc}}(5)$ & $15.1^{\mathrm{c}}(3)$ \\
Ketones & $74.6^{\mathrm{b}}(6)$ & $141^{\mathrm{a}}(4)$ & $5.5^{\mathrm{c}}(4)$ & $\mathrm{nd}^{*}$ \\
Alcohols & $82.8^{\mathrm{a}}(8)$ & $70.6^{\mathrm{a}}(6)$ & $8.7^{\mathrm{c}}(2)$ & $39.1^{\mathrm{b}}(5)$ \\
Aromatic compounds & $373.2^{\mathrm{a}}(20)$ & $136.3^{\mathrm{b}}(15)$ & $144.5^{\mathrm{b}}(23)$ & $7.7^{\mathrm{c}}(5)$ \\
Esters & $72.8^{\mathrm{a}}(3)$ & $61.2^{\mathrm{b}}(4)$ & $23.8^{\mathrm{c}}(2)$ & $4.9^{\mathrm{d}}(3)$ \\
Furans & $35.1^{\mathrm{ab}}(2)$ & $19.3^{\mathrm{ab}}(3)$ & $45.2^{\mathrm{a}}(2)$ & $7.9^{\mathrm{c}}(3)$ \\
Alkanes & $32.2^{\mathrm{a}}(3)$ & $5.4^{\mathrm{b}}(2)$ & $25.8^{\mathrm{ab}}(2)$ & $\mathrm{nd}$ \\
Alkenes & $24.1^{\mathrm{a}}(3)$ & $12^{\mathrm{b}}(2)$ & $9.1^{\mathrm{b}}(2)$ & $\mathrm{nd}$ \\
Terpenes & $717.2^{\mathrm{a}}(9)$ & $275.1^{\mathrm{c}}(7)$ & $451.5^{\mathrm{b}}(11)$ & $17^{\mathrm{d}}(5)$ \\
Others & $69^{\mathrm{b}}(1)$ & $202.9^{\mathrm{a}}(5)$ & $16^{\mathrm{bc}}(2)$ & $11.4^{\mathrm{c}}(5)$ \\
Total compounds & $2039.4^{\mathrm{a}}(65)$ & $1267.6^{\mathrm{b}}(56)$ & $1002.1^{\mathrm{bc}}(55)$ & $103.4^{\mathrm{cd}}(29)$ \\
\hline
\end{tabular}

${ }^{\text {abcd }}$ Values reported are the mean of two replicates. Mean values for each volatile compound in the same row followed by different letters differ significantly $(p<0.05) .{ }^{*}$ nd: not detected. 
Table 3. Results of the factorial design for the optimization of the extraction conditions of volatile compounds in tiger nut beverages.

\begin{tabular}{|c|c|c|c|c|c|c|}
\hline \multirow{2}{*}{ Compounds } & \multicolumn{3}{|c|}{ Factor } & \multicolumn{3}{|c|}{ Significant interaction } \\
\hline & Vol $^{\mathrm{b}}$ & $\mathrm{NaCl}^{\mathrm{c}}$ & Temp $^{d}$ & $\mathrm{Vol}^{*} \mathrm{NaCl}$ & Vol* temp & $\mathrm{NaCl}^{*}$ temp \\
\hline 2-propanone & & Yes & & & & ${ }^{*} \mathrm{e}$ \\
\hline Propyl acetate & & Yes & & & ${ }^{*}$ & \\
\hline \multicolumn{7}{|l|}{ Alpha-pinene } \\
\hline Sabinene & & No & 60 & & & \\
\hline Heptanal & & No & 60 & ** & $*$ & \\
\hline Limonene & & No & 60 & & & $* * \mathrm{f}$ \\
\hline Propyl benzene & & No & 60 & & & \\
\hline 1-hexanol & 2 & Yes & & ** & $*$ & ** \\
\hline Nonanal & & Yes & 60 & & & ** \\
\hline Benzaldehyde & 2 & Yes & 60 & $* *$ & $*$ & \\
\hline Geranial & & & 60 & & & \\
\hline Benzeneethanol & & Yes & 60 & & & ** \\
\hline Eugenol & & Yes & 60 & * & & \\
\hline 3-octanol & & No & 60 & * & & \\
\hline Linalool & & No & 60 & $*$ & & $* *$ \\
\hline \multicolumn{7}{|l|}{ Myrceme } \\
\hline 2-3-dihydroindene & & No & 60 & & * & $* *$ \\
\hline Octane & & Yes & 40 & $*$ & & $* *$ \\
\hline Ethanol & & Yes & 40 & & & ** \\
\hline Chloroform & & Yes & 40 & & & \\
\hline 1-decene & & No & 60 & & & * \\
\hline Toluene & & No & & & & \\
\hline Hexanal & 2 & Yes & & $* *$ & * & \\
\hline \multicolumn{7}{|l|}{ Beta-pinene } \\
\hline Benzene-ethyl & & No & & & & \\
\hline Benzene-1,4-dimethyl & & No & 60 & & & \\
\hline p-xylene & & No & & & & \\
\hline 2-heptanone & & No & 60 & & * & \\
\hline Alpha-terpinene & 4 & No & & $*$ & $* *$ & * \\
\hline Benzene-1-ethyl-3-methyl & & No & 60 & & & $* *$ \\
\hline Gamma-terpinene & 4 & No & 60 & * & & $* *$ \\
\hline Benzene-1,2,3-trimethyl & & No & & & & \\
\hline Benzene-1-ethyl-2-mehyl & & No & 60 & & & ** \\
\hline Terpinolene & & No & & & & \\
\hline
\end{tabular}




\section{Continued}

\begin{tabular}{|c|c|c|c|c|c|c|}
\hline Benzene-1,2,4-trimethyl & & No & & & & \\
\hline Benzene-1-methyl-3-propyl & & No & 60 & & & \\
\hline Benzene-4-ethyl-1,2-dimethyl & & No & 60 & & & \\
\hline \multicolumn{7}{|l|}{ 6-methyl-5-hepten-2-one } \\
\hline Benzene-2-ethyl-1,4-dimethyl & 2 & Yes & 60 & ** & ** & ** \\
\hline Benzene-1-methyl-4,1-methyl & & No & 60 & & & * \\
\hline Benzene-1-ethyl-1,3-dimethyl & & No & & & & \\
\hline 2 nonanone & & No & 60 & ** & * & ** \\
\hline 2-ethy-3-methyl-pyrazine & 2 & Yes & 60 & ** & & ** \\
\hline Benzene-1,2,3,5-tetramethyl & & & 60 & * & & \\
\hline Heptanol & 2 & & 60 & ** & & \\
\hline \multicolumn{7}{|l|}{ 2-butanone } \\
\hline 2-decanona & & No & 60 & & & ** \\
\hline Beta-caryophyllene & 2 & Yes & 60 & & & \\
\hline Nonanol & & No & 60 & & & ** \\
\hline \multicolumn{7}{|l|}{ 2-furanmethanol } \\
\hline 2cyclohexen-1-ol-4,4,6-trimethyl & 2 & & 60 & ** & & \\
\hline Alpha-terpineol & 2 & & 60 & ** & & * \\
\hline Cyclododecane & & No & 60 & & & ** \\
\hline Geranyl propionate & & & 60 & & & \\
\hline Azulene & & No & 60 & & & ** \\
\hline Benzaldehyde propylene glycol acetal & 2 & Yes & 60 & ** & & \\
\hline Phenol 2 methoxy & 2 & Yes & 60 & ** & & ** \\
\hline Ethanone & & Yes & & & & \\
\hline Phenol & 2 & & & & & \\
\hline Phenol-4-ethyl-2-methoxy & 2 & Yes & 60 & ** & & $*$ \\
\hline Hydroxynonanonic acid lactona & 2 & Yes & 60 & ** & * & ** \\
\hline 2-propenal-3-phenyl & & Yes & 60 & & & \\
\hline 4-vinyl-2-methoxy-phenol & & Yes & 60 & & & ** \\
\hline Benzoic acid-2-amino-methyl & & Yes & 60 & ** & & * \\
\hline
\end{tabular}

${ }^{a}$ The significance of each factor and interaction was tested by ANOVA. Only significant factors $(p<0.05)$ are reported. The selection of the optimal values was achieved by selecting the LSMEANS with a greater response. ${ }^{b}$ Volume $(2 \mathrm{~mL}$ or $4 \mathrm{~mL})$. ${ }^{c} \mathrm{NaCl}$ presence or absence (yes or no). ${ }^{\mathrm{d}} \mathrm{Temperature}\left(40^{\circ} \mathrm{C}\right.$ or $\left.60^{\circ} \mathrm{C}\right)$. ${ }^{\mathrm{e}}$ Significant interaction between factors $(p<0.05)$. ${ }^{\mathrm{f}}$ Significant interaction between factors $(p<0.01)$.

benzaldehyde, that may prevent other less abundant volatiles from being extracted due to the competition of fiber sites. Furthermore, reverse diffusion from the fiber to the sample has been reported previously due to overloading of the fiber. Also it has been reported that if a sample volume is large and the extraction time is lengthened, desorption phenomenon could occur [15]. This could be the case for some compounds since our analysis was conducted for $60 \mathrm{~min}$. 
Of all the variables identified, $\sim 80 \%$ of them were affected by the addition of salt. Salts are often added to aqueous samples to increase the concentration of the aroma compounds in the vapor phase [16]. However, the majority ( $56 \%$ ) of the variables, which had some type of effect with the addition of salt in our design, did not show increased sensitivity with salt saturation.

Taking into account the results described, the best extraction conditions for commercial tiger nut beverages using a DVB/CAR/PDMS fiber were $2 \mathrm{~mL}$ of sample volume, without addition of $\mathrm{NaCl}$ and extraction temperature at $60^{\circ} \mathrm{C}$.

\subsection{Volatile Compounds of Tiger Nut Milk}

A total of 83 compounds, characterized by different functionality, were detected in the volatile fraction of tiger nut beverages, including 14 aldehydes, 14 alcohols, 8 ketones, 11 aromatic compounds, 7 esters, 4 furans, 6 alkanes/alkenes and 19 terpenes (Table 4). Among the detected compounds, 7 were present in all the analyzed tiger nut samples, namely ethanol, 1-nonanol, 4-vinyl-2-methoxy-phenol, alpha-pinene, sabinene, limonene and alpha-terpinolene (Table 5). Among the 9 commercial samples analyzed, the pasteurized sample (OXT9) showed the fewest number of compounds identified in headspace. This result could be related to lack of ingredients such as flavourings or preservatives which comprise the production of all other samples analyzed (Table 1).

Table 4. Volatile compounds detected in the headspace of tiger nut beverages.

\begin{tabular}{|c|c|c|c|c|c|}
\hline Compounds & $\mathbf{R T}^{\mathbf{a}}$ & KI exp..$^{b}$ & KI lit. ${ }^{c}$ & ID $^{d}$ & Percept $^{\text {h }}$ \\
\hline \multicolumn{6}{|c|}{ Aldehydes } \\
\hline Pentanal & 9.138 & 989 & $998.1^{\mathrm{j}}$ & $\mathrm{MS}^{\mathrm{e}}, \mathrm{IR}^{\mathrm{f}}, \mathrm{S}^{\mathrm{g}}$ & Pungent, almond-like, herbaceous, green, malty, rubber \\
\hline n-heptanal & 13.426 & 1195 & $1199.6^{\mathrm{j}}$ & MS, IR & $\begin{array}{l}\text { Citrus fruit, green, fatty, dry fish, pesticide, solvent, } \\
\text { smoky, rancid, fruity }\end{array}$ \\
\hline n-hexanal & 11.384 & 1091 & $1098.0^{\mathrm{j}}$ & MS, IR, S & $\begin{array}{l}\text { Green, fruity, acorn-like, tallowy, fishy, grassy, herbal, } \\
\text { leafy }\end{array}$ \\
\hline Decanal & 18.326 & 1517 & $1505^{\mathrm{k}}$ & MS, IR, S & $\begin{array}{l}\text { Stewed, burned, gree, waxy, orangeskin-like, floral, } \\
\text { lemon, fatty, herbaceous, soapy }\end{array}$ \\
\hline Octanal & 15.014 & 1289 & $1299^{\mathrm{k}}$ & MS, IR, S & $\begin{array}{l}\text { Lemon, stew-like, boiled meat-like, rancid, soapy, } \\
\text { citrus, green, flower, fruit, orange }\end{array}$ \\
\hline Trans-2-decenal & 20.261 & 1670 & $1651^{\mathrm{m}}$ & MS, IR & Green, fatty, tallowy, orange-like \\
\hline 2-propenal-3-phenyl & 23.351 & 1944 & $2017^{\mathrm{s}}$ & MS, IR & \\
\hline Benzaldehyde & 18.889 & 1560 & $1534^{\mathrm{i}}$ & MS, IR, S & Burnt sugar, almond, woody \\
\hline Trans-2-heptenal & 15.882 & 1345 & $1332^{\mathrm{n}}$ & MS, IR & $\begin{array}{l}\text { Sulfury, grassy, fatty, almond-like, pesticide, onion, } \\
\text { mushroom, earthy }\end{array}$ \\
\hline Nonanal & 16.661 & 1396 & $1394^{\mathrm{i}}$ & MS, IR, S & Floral, waxy, melon, soapy, fatty, lavender, citrus fruit \\
\hline 4-methoxybenzaldehyde & 24.148 & 2015 & 2051 & MS, IR & Mint-like, sweet \\
\hline Trans-cinnamaldehyde & 25.088 & 2091 & $2017^{\mathrm{s}}$ & MS, IR & \\
\hline Trans,trans-2,4-decadienal & 22.261 & 1844 & $1778^{\circ}$ & MS, IR & Fatty, waxy, deep-fried, pungent, green, citrus \\
\hline Perilladehyde & 22.177 & 1837 & $1777^{\mathrm{s}}$ & MS, IR & \\
\hline \multicolumn{6}{|c|}{ Ketones } \\
\hline 6-methyl-5-hepten-2-one & 16.014 & 1354 & $1342^{\mathrm{i}}$ & MS, IR, S & $\begin{array}{l}\text { Mushroom, earthy, vinyl, rubber, woody, blackcurrant, } \\
\text { boiled fruit }\end{array}$ \\
\hline 2-nonanone & 16.775 & 1403 & 14361 & MS, IR, S & Fruity, soapy, fatty, green, earthy, baked \\
\hline 2-decanone & 18.242 & 1510 & $1497^{\mathrm{s}}$ & MS, IR & Fruity, soapy, fatty, green, earthy, baked \\
\hline 2-butanone & 18.338 & 1517 & & MS & Chocolate, chemical, gas, ethereal, cheese, butter \\
\hline
\end{tabular}




\section{Continued}

\begin{tabular}{|c|c|c|c|c|c|}
\hline 2-heptanone & 13.384 & 1193 & $1195.8^{j}$ & MS, IR, S & $\begin{array}{l}\text { Cured ham-like, toasted, nutty, gas, gravy, soapy, } \\
\text { fruity }\end{array}$ \\
\hline 3-octanone & 14.63 & 1266 & $1265.5^{\mathrm{j}}$ & MS, IR & Herbal, buttery \\
\hline 2-propanone & 5.825 & 825 & $814^{\mathrm{s}}$ & MS, IR, S & Glue, fruity \\
\hline 1-phenylethanone & 20.542 & 1693 & $1650^{\mathrm{s}}$ & MS, IR & Cheesy, sweet, almond-like, floral \\
\hline \multicolumn{6}{|c|}{ Alcohols } \\
\hline 2-heptanol & 15.541 & 1322 & $1319^{\mathrm{i}}$ & MS, IR, S & $\begin{array}{c}\text { Mushroom-like, herbaceous, pungent, acrid, roquefort } \\
\text { cheese, like, fruity }\end{array}$ \\
\hline 1-pentanol & 14.487 & 1258 & $1255^{\mathrm{i}}$ & MS, IR, S & Fruity, green, sweet, pungent \\
\hline Benzeneethanol & 23.345 & 1943 & $1940^{\mathrm{i}}$ & MS, IR & $\begin{array}{l}\text { Honey-like, sweet, yeast-like, floral, spicy, herbal, } \\
\text { rose, pollen }\end{array}$ \\
\hline Benzenemethanol & 22.92 & 1904 & $1927^{\mathrm{s}}$ & MS, IR & Aromatic, floral, fruity \\
\hline Ethanol & 8.054 & 942 & $936^{\mathrm{s}}$ & MS, IR, S & Ethanol-like, pungent, sweet \\
\hline 1-octen-3-ol & 17.445 & 1452 & $1451^{\mathrm{i}}$ & MS, IR, S & $\begin{array}{l}\text { Garlic, mushroom, spicy, rubbery, carrots, herbaceous, } \\
\text { dirty, dust, earthy }\end{array}$ \\
\hline 1-heptanol & 17.571 & 1462 & $1455^{\mathrm{i}}$ & MS, IR & Fresh, light green, nutty \\
\hline 1-hexanol & 16.194 & 1366 & $1355^{\mathrm{i}}$ & MS, IR, S & $\begin{array}{l}\text { Flowery, toasty, dry, fruity, herbal, mild woody, sweet, } \\
\text { green grass, leafy }\end{array}$ \\
\hline 1-octanol & 18.913 & 1562 & $1565^{\mathrm{s}}$ & MS, IR, S & $\begin{array}{l}\text { Metallic, sulfur, burnt matches, toasted bread, herbal, } \\
\text { fatty, green, floral }\end{array}$ \\
\hline Phenol-2-methoxy & 22.812 & 1894 & 1883 & MS, IR & $\begin{array}{c}\text { Smokey, sweet, phenolic, chemical, green, fatty, } \\
\text { medicine }\end{array}$ \\
\hline 1-nonanol & 20.153 & 1662 & $1661^{\mathrm{i}}$ & MS, IR & Fat, green, fruity \\
\hline Phenol & 24.316 & 2029 & $2209^{1}$ & MS, IR, S & Phenolic, medicinal odor \\
\hline 4-vinyl-2-methoxy-phenol & 26.987 & 2229 & $2223^{\mathrm{s}}$ & MS, IR & Clove-like, phenolic, smokey \\
\hline z-isoeugenol & 29.7 & 2380 & $2298^{\mathrm{s}}$ & MS, IR & \\
\hline \multicolumn{6}{|c|}{ Aromatic compounds } \\
\hline Ethylbenzene & 12.18 & 1132 & $1124^{\mathrm{m}}$ & MS, IR, S & Ethereal, floral, sweet \\
\hline p-xylene & 12.33 & 1140 & $1150^{\mathrm{s}}$ & MS, IR, S & Plastic, cold meat fat-like \\
\hline n-propyl-benzene & 13.63 & 1206 & $1207^{\mathrm{s}}$ & MS, IR & \\
\hline 1-ethyl 2-methylbenzene & 14.109 & 1236 & $1257^{\mathrm{s}}$ & MS, IR & \\
\hline 1,2,4-trimethylbenzene & 14.876 & 1281 & $1293^{\mathrm{s}}$ & MS, IR & Plastic, herbaceous \\
\hline p-cymene & 14.936 & 1284 & $1285^{\mathrm{k}}$ & MS, IR, S & Lemon, fruity, fuel-like, sweet, herbal, spicy \\
\hline 2,3-dihydroindene & 16.715 & 1399 & & MS & \\
\hline Toluene (benzene -methyl) & 10.551 & 1055 & $1042^{\mathrm{s}}$ & MS, IR, S & $\begin{array}{c}\text { Pungent, caramel, ethereal, synthetic, fruity, rubbery, } \\
\text { solvent-like }\end{array}$ \\
\hline n-butylbenzene & 15.463 & 1317 & $1308^{\mathrm{s}}$ & MS, IR & \\
\hline m-cymene & 16.265 & 1370 & $1267^{\mathrm{s}}$ & MS, IR & Lemon, fruity, fuel-like, sweet, herbal, spicy \\
\hline Styrene & 14.78 & 1275 & $1273^{\mathrm{s}}$ & MS, IR & Pungent, aromatic, fragrant, roasty \\
\hline \multicolumn{6}{|c|}{ Esters } \\
\hline $\begin{array}{l}\text { Cinnamaldehyde propylene } \\
\text { glycol acetal }\end{array}$ & 28.203 & 2298 & & MS & \\
\hline Ethyl hexadecanoate & 27.49 & 2258 & $2229^{\mathrm{s}}$ & MS, IR & Waxy, mild sweet \\
\hline $\begin{array}{l}\text { Benzaldehyde propylene } \\
\text { glycol acetal }\end{array}$ & 22.686 & 1883 & & MS & \\
\hline
\end{tabular}




\section{Continued}

\begin{tabular}{|c|c|c|c|c|c|}
\hline Geranyl acetate & 21.369 & 1765 & $1760^{\mathrm{s}}$ & MS, IR & Floral, rose, sweet, fruity \\
\hline Propyl acetate & 9.048 & 985 & & MS & \\
\hline \multicolumn{6}{|c|}{ Furans } \\
\hline Furan-2-pentyl & 13.768 & 1215 & $1244^{\mathrm{q}}$ & MS, IR, S & Buttery, green bean-like \\
\hline 2-furancarboxaldehyde & 17.996 & 1492 & & MS & \\
\hline 2-furanmethanol & 20.303 & 1674 & $1669^{\mathrm{s}}$ & MS, IR & Weak, fermented, burnt sugar, creamy, caramellic note \\
\hline $\begin{array}{l}\text { 2(3H)furanone } \\
\text { dihydro-5-phenyl }\end{array}$ & 24.909 & 2077 & & MS & \\
\hline \multicolumn{6}{|c|}{ Alkanes/Alkenes } \\
\hline Octane & 5.472 & 802 & $800^{\mathrm{s}}$ & MS, IR, S & Fusel-like, fruity, sweet \\
\hline Decane & 9.419 & 1000 & $1000^{\mathrm{s}}$ & MS, IR, S & Fusel-like, fruity, sweet \\
\hline n-tetradecane & 16.643 & 1394 & $1399^{\mathrm{m}}$ & MS, IR & Mild herbaceous, sweet, fusel-like \\
\hline Dodecane & 13.265 & 1187 & $1200^{\mathrm{s}}$ & MS, IR, S & Fusel-like \\
\hline Tetradecene & 17.344 & 1445 & $1400^{\mathrm{s}}$ & MS, IR, S & Green \\
\hline (e,e)-2,4-hexadiene & 5.478 & 803 & & MS & \\
\hline \multicolumn{6}{|c|}{ Terpenes } \\
\hline Alpha-pinene & 10.018 & 1030 & $1032^{\mathrm{q}}$ & MS, IR & $\begin{array}{l}\text { Terpeny, fruity, sweet, green, woody, pine, citrus, lime, } \\
\text { camphoraceous }\end{array}$ \\
\hline Alpha-thujene & 10.132 & 1035 & $1038^{\mathrm{s}}$ & MS, IR & Woody, herbal, green \\
\hline Camphene & 10.977 & 1074 & $1077^{\mathrm{s}}$ & MS, IR & Sweet, fruity, camphoraceous, pine, oily, herb \\
\hline Beta-pinene & 11.737 & 1108 & $1113^{\mathrm{s}}$ & MS, IR & Musty, green, sweet, pine, resin, turpentine, woody \\
\hline Sabinene & 11.959 & 1120 & $1123^{\mathrm{s}}$ & MS, IR & Fresh, citrus note, spicy, seet, woody \\
\hline Myrcene & 12.618 & 1155 & $1168^{\mathrm{s}}$ & MS, IR & $\begin{array}{l}\text { Metallic, musty, geranium, sweet, fruity, ethereal, } \\
\text { soapy, lemon, spicy, woody }\end{array}$ \\
\hline Limonene & 13.271 & 1187 & $1203^{\mathrm{q}}$ & MS, IR, S & Licorice, green, citrus-like, ethereal, fruity \\
\hline Alpha-phellandrene & 12.702 & 1159 & $1205^{\mathrm{s}}$ & MS, IR & Fruity, minty, herbaceous, citrus, lime, pepper, juniper \\
\hline Beta-pinene & 13.42 & 1195 & $1124^{\mathrm{s}}$ & MS, IR & Musty, green, sweet, pine, resin, turpentine, woody \\
\hline Alpha-terpinolene & 14.684 & 1270 & $1275^{\mathrm{s}}$ & MS, IR & Woody, fruity, sweet, piney, slightly anisic \\
\hline Alpha-terpinene & 12.935 & 1171 & $1178^{\mathrm{s}}$ & MS, IR & Gasoline-like, ethereal, fruity, lemon \\
\hline Gamma-terpinene & 14.457 & 1256 & $1262^{\mathrm{s}}$ & MS, IR & Citrus-like, terpeny, herbaceous, fruity, sweet \\
\hline Linalool & 18.757 & 1550 & $155^{\mathrm{s}}$ & MS, IR, S & $\begin{array}{c}\text { Muscat, sweet, green, floral, lemon, parsley, laven- } \\
\text { der-like, fruity }\end{array}$ \\
\hline Beta-terpineol & 19.985 & 1648 & $1640^{\mathrm{s}}$ & MS, IR & Pungent, woody, earthy \\
\hline Eugenol & 26.544 & 2203 & $2192^{\mathrm{s}}$ & MS, IR & $\begin{array}{l}\text { Spicy, honey, clove, balsamic, herbaceous, cam- } \\
\text { phoraceous }\end{array}$ \\
\hline Terpinen-4-ol & 19.716 & 1626 & $1616^{\mathrm{s}}$ & MS, IR & $\begin{array}{l}\text { Terpeny, woody, sweet, herbaceous, pine, musty, } \\
\text { fruity, licorices, moldy }\end{array}$ \\
\hline Trans-isoeugenol & 27.951 & 2284 & & MS & \\
\hline Eucalyptol & 13.828 & 1219 & $1214^{\mathrm{s}}$ & MS, IR & $\begin{array}{l}\text { Camphoraceous, minty, sweet, liquorices, menthol, } \\
\text { pine }\end{array}$ \\
\hline Alpha-terpineol & 20.812 & 1716 & $1719^{\mathrm{s}}$ & MS, IR & Peach-like, anise, oily, fruity, floral, minty, toothpaste \\
\hline
\end{tabular}

${ }^{\mathrm{a}} \mathrm{RT}$ : Retention time (minutes). ${ }^{\mathrm{b}} \mathrm{KI}$ exp.: linear retention indexes experimentally calculated under the set chromatographic conditions. ${ }^{\mathrm{c}} \mathrm{KI}$ lit.: linear retention indexes reported in the literature. ${ }^{\mathrm{d}}$ Identification method. ${ }^{\mathrm{e}} \mathrm{MS}$ : Tentatively identified by mass spectra (comparison with Wiley library). ${ }^{\mathrm{f}} \mathrm{IR}$ : Tentatively identified by retention index. ${ }^{\mathrm{g}}$ Identified by standars. ${ }^{\mathrm{h}}$ Characteristic odor as reported in Pherobase and Flavornet databases: ${ }^{\mathrm{i}}$ [25], ${ }^{\mathrm{j}}$ [26], ${ }^{\mathrm{k}}[27],{ }^{\mathrm{l}}[28],{ }^{\mathrm{n}}[29],{ }^{\mathrm{o}}[30],{ }^{\mathrm{q}}[31],{ }^{\mathrm{s}}[32]$ and [33]. 
Table 5. Volatile compounds identified in different types of Tiger nut milk after HS-SPME using DVB/CAR/PDMS fiber coating and GC-MS analysis (extraction temperature: $60^{\circ} \mathrm{C}$; sample with $2 \mathrm{~mL}$; extraction time: $60 \mathrm{~min}$ ). The results were expressed as $\mu \mathrm{g}$ of 4 methyl-2-pentanol/mL of tiger nut beverage.

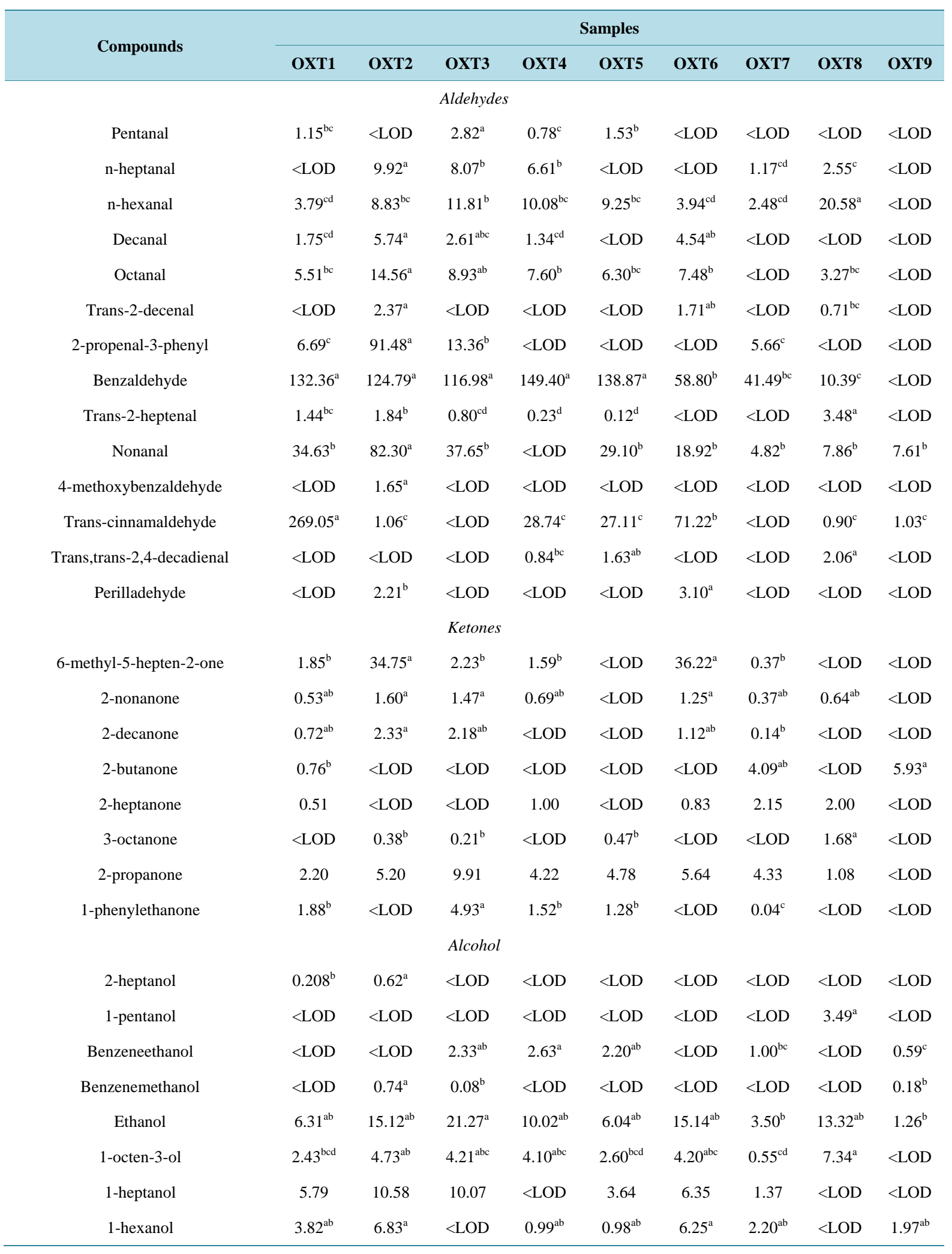




\section{Continued}

\begin{tabular}{|c|c|c|c|c|c|c|c|c|c|}
\hline \\
\hline 1-octanol & $<\mathrm{LOD}$ & $<\mathrm{LOD}$ & $<\mathrm{LOD}$ & $<\mathrm{LOD}$ & $<\mathrm{LOD}$ & $<\mathrm{LOD}$ & $<\mathrm{LOD}$ & $<\mathrm{LOD}$ & $6.30^{\mathrm{a}}$ \\
\hline Phenol-2-methoxy & 0.50 & 0.67 & $<\mathrm{LOD}$ & $<\mathrm{LOD}$ & 0.45 & $<\mathrm{LOD}$ & 0.23 & $<\mathrm{LOD}$ & $<\mathrm{LOD}$ \\
\hline 1-nonanol & $10.09^{\mathrm{ab}}$ & $15.55^{\mathrm{a}}$ & $7.77^{\mathrm{ab}}$ & $9.98^{\mathrm{ab}}$ & $10.17^{\mathrm{ab}}$ & $7.44^{\mathrm{ab}}$ & $1.85^{\mathrm{b}}$ & $3.94^{\mathrm{ab}}$ & $2.00^{\mathrm{b}}$ \\
\hline Phenol & $<\mathrm{LOD}$ & $<\mathrm{LOD}$ & $0.74^{\mathrm{a}}$ & $<\mathrm{LOD}$ & $<\mathrm{LOD}$ & $<\mathrm{LOD}$ & $0.31^{\mathrm{c}}$ & $0.50^{\mathrm{b}}$ & $0.83^{\mathrm{a}}$ \\
\hline 4-vinyl-2-methoxy-phenol & 5.63 & 13.53 & 7.48 & 3.19 & 2.07 & 8.51 & 0.63 & 7.38 & 1.02 \\
\hline 4-ethyl-2-methoxyphenol & 3.06 & $<\mathrm{LOD}$ & 2.01 & 2.10 & $<\mathrm{LOD}$ & 2.98 & 0.28 & $<\mathrm{LOD}$ & 0.68 \\
\hline z-isoeugenol & $8.68^{\mathrm{a}}$ & $2.65^{\mathrm{b}}$ & $1.53^{\mathrm{bc}}$ & $<\mathrm{LOD}$ & $<\mathrm{LOD}$ & $<\mathrm{LOD}$ & $<\mathrm{LOD}$ & $<$ LOD & $0.60^{\text {cd }}$ \\
\hline \multicolumn{10}{|c|}{ Aromatic } \\
\hline Ethylbenzene & 1.32 & 2.84 & 1.23 & 4.98 & $<\mathrm{LOD}$ & 4.15 & $<\mathrm{LOD}$ & $<$ LOD & 1.19 \\
\hline p-xylene & 0.45 & 9.29 & 4.21 & $<\mathrm{LOD}$ & 3.32 & 5.90 & 1.81 & 3.47 & 1.74 \\
\hline n-propyl-benzene & $<\mathrm{LOD}$ & $<\mathrm{LOD}$ & $<\mathrm{LOD}$ & $2.96^{\mathrm{a}}$ & $3.32^{\mathrm{a}}$ & $<\mathrm{LOD}$ & $2.21^{\mathrm{b}}$ & $<$ LOD & $<\mathrm{LOD}$ \\
\hline 1-ethyl 2-methylbenzene & $<\mathrm{LOD}$ & 1.39 & 1.58 & 16.26 & 5.59 & 3.85 & $<\mathrm{LOD}$ & $<$ LOD & $<$ LOD \\
\hline 1,2,4-trimethylbenzene & $1.41^{\mathrm{b}}$ & $4.02^{\mathrm{b}}$ & $6.41^{\mathrm{b}}$ & $29.35^{\mathrm{a}}$ & $6.16^{\mathrm{b}}$ & $6.47^{\mathrm{b}}$ & $<\mathrm{LOD}$ & $0.50^{\mathrm{b}}$ & $1.58^{\mathrm{b}}$ \\
\hline p-cymene & $58.28^{\mathrm{a}}$ & $33.47^{\mathrm{abc}}$ & $18.03^{\mathrm{bcd}}$ & $10.04^{\mathrm{cd}}$ & $3.87^{\mathrm{d}}$ & $39.08^{\mathrm{ab}}$ & $<\mathrm{LOD}$ & $<$ LOD & $32.63^{\mathrm{abc}}$ \\
\hline 2,3-dihydroindene & $0.54^{\text {cd }}$ & $0.63^{\mathrm{bc}}$ & $0.84^{\mathrm{bc}}$ & $2.55^{\mathrm{a}}$ & $<\mathrm{LOD}$ & $1.21^{\mathrm{b}}$ & $2.09^{\mathrm{a}}$ & $<$ LOD & $<$ LOD \\
\hline Toluene (benzene -methyl) & $0.96^{\mathrm{bc}}$ & $2.77^{\mathrm{abc}}$ & $2.58^{\mathrm{abc}}$ & $3.33^{\mathrm{abc}}$ & $2.82^{\mathrm{abc}}$ & $3.85^{\mathrm{ab}}$ & $<$ LOD & $3.95^{\mathrm{ab}}$ & $4.44^{\mathrm{a}}$ \\
\hline n-butylbenzene & $<\mathrm{LOD}$ & $<\mathrm{LOD}$ & $<\mathrm{LOD}$ & $0.75^{\mathrm{a}}$ & $0.61^{\mathrm{a}}$ & $<\mathrm{LOD}$ & $<\mathrm{LOD}$ & $<$ LOD & $<\mathrm{LOD}$ \\
\hline m-cymene & $1.09^{\mathrm{a}}$ & $<\mathrm{LOD}$ & $<$ LOD & $<$ LOD & $<\mathrm{LOD}$ & $1.59^{\mathrm{a}}$ & $<\mathrm{LOD}$ & $<$ LOD & $<$ LOD \\
\hline Styrene & $<\mathrm{LOD}$ & $13.15^{\mathrm{a}}$ & $1.13^{\mathrm{bc}}$ & $2.94^{\mathrm{bc}}$ & $4.41^{\mathrm{bc}}$ & $12.38^{\mathrm{a}}$ & $<\mathrm{LOD}$ & $4.66^{\mathrm{b}}$ & $9.96^{\mathrm{a}}$ \\
\hline \multicolumn{10}{|c|}{ Ester } \\
\hline Cinnamaldehyde propylene glycol acetal & $33.58^{\mathrm{a}}$ & $<\mathrm{LOD}$ & $3.47^{\mathrm{b}}$ & $3.69^{\mathrm{b}}$ & $1.14^{\mathrm{b}}$ & $<\mathrm{LOD}$ & $<\mathrm{LOD}$ & $<$ LOD & $<$ LOD \\
\hline Ethyl hexadecanoate & $0.34^{\mathrm{bc}}$ & $0.66^{\mathrm{ab}}$ & $<\mathrm{LOD}$ & $<\mathrm{LOD}$ & $0.85^{\mathrm{ab}}$ & $<\mathrm{LOD}$ & $<\mathrm{LOD}$ & $0.62^{\mathrm{ab}}$ & $1.05^{\mathrm{a}}$ \\
\hline Benzaldehyde propylene glycol acetal & $12.27^{\mathrm{c}}$ & $<\mathrm{LOD}$ & $17.12^{\mathrm{bc}}$ & $54.23^{\mathrm{a}}$ & $24.53^{b}$ & $<\mathrm{LOD}$ & $8.28^{\mathrm{cd}}$ & $<$ LOD & $<$ LOD \\
\hline Geranyl acetate & $4.78^{\mathrm{bc}}$ & $12.93^{\mathrm{ab}}$ & $1.27^{\mathrm{c}}$ & $<\mathrm{LOD}$ & $1.75^{\mathrm{c}}$ & $17.97^{\mathrm{a}}$ & $0.53^{\mathrm{c}}$ & $<$ LOD & $<$ LOD \\
\hline Propyl acetate & $<$ LOD & $<\mathrm{LOD}$ & $<\mathrm{LOD}$ & $<\mathrm{LOD}$ & $5.05^{\mathrm{a}}$ & $<\mathrm{LOD}$ & $1.73^{\mathrm{ab}}$ & $<$ LOD & $<$ LOD \\
\hline \multicolumn{10}{|c|}{ Furan } \\
\hline Furan-2-pentyl & 9.45 & 15.82 & 10.64 & 11.94 & 24.18 & 18.23 & $<\mathrm{LOD}$ & 14.27 & $<\mathrm{LOD}$ \\
\hline 2-furancarboxaldehyde & $1.37^{\mathrm{bc}}$ & $<\mathrm{LOD}$ & $12.49^{\mathrm{a}}$ & $<\mathrm{LOD}$ & $10.79^{\mathrm{ab}}$ & $<\mathrm{LOD}$ & $<\mathrm{LOD}$ & $0.73^{\mathrm{c}}$ & $14.31^{\mathrm{a}}$ \\
\hline 2-furanmethanol & $<\mathrm{LOD}$ & $<\mathrm{LOD}$ & $7.30^{\mathrm{a}}$ & $4.68^{\mathrm{ab}}$ & $<\mathrm{LOD}$ & $<\mathrm{LOD}$ & $2.02^{\mathrm{ab}}$ & $<\mathrm{LOD}$ & $4.96^{\mathrm{ab}}$ \\
\hline Furan-2-heptyl & $<$ LOD & $1.88^{\mathrm{a}}$ & $<\mathrm{LOD}$ & $<\mathrm{LOD}$ & $<\mathrm{LOD}$ & $0.87^{\mathrm{ab}}$ & $<\mathrm{LOD}$ & $<$ LOD & $<\mathrm{LOD}$ \\
\hline 2(3h)furanone dihydro-5-phenyl & $5.82^{\mathrm{a}}$ & $3.26^{\mathrm{b}}$ & $<\mathrm{LOD}$ & $<\mathrm{LOD}$ & $<\mathrm{LOD}$ & $<\mathrm{LOD}$ & $<\mathrm{LOD}$ & $<$ LOD & $<$ LOD \\
\hline \multicolumn{10}{|c|}{ Alkane/alkene } \\
\hline Octane & 0.47 & 2.89 & 0.48 & $<\mathrm{LOD}$ & 0.83 & 0.99 & 0.06 & 1.23 & $<\mathrm{LOD}$ \\
\hline Decane & $<\mathrm{LOD}$ & $<\mathrm{LOD}$ & $6.31^{\mathrm{a}}$ & $2.83^{\mathrm{ab}}$ & $<\mathrm{LOD}$ & $<\mathrm{LOD}$ & $4.79^{\mathrm{ab}}$ & $<\mathrm{LOD}$ & $<\mathrm{LOD}$ \\
\hline n-tetradecane & $<\mathrm{LOD}$ & $<$ LOD & 2.57 & 1.80 & 4.02 & 5.77 & $<\mathrm{LOD}$ & $<$ LOD & $<\mathrm{LOD}$ \\
\hline Dodecane & $<\mathrm{LOD}$ & $0.16^{\mathrm{b}}$ & $17.53^{\mathrm{a}}$ & $<\mathrm{LOD}$ & $<\mathrm{LOD}$ & $<\mathrm{LOD}$ & $<\mathrm{LOD}$ & $0.12^{\mathrm{b}}$ & $<\mathrm{LOD}$ \\
\hline
\end{tabular}




\section{Continued}

\begin{tabular}{|c|c|c|c|c|c|c|c|c|c|}
\hline Tetradecene & $<\mathrm{LOD}$ & $<$ LOD & $2.61^{\mathrm{a}}$ & $<\mathrm{LOD}$ & $<\mathrm{LOD}$ & $<\mathrm{LOD}$ & $<\mathrm{LOD}$ & $<\mathrm{LOD}$ & $<\mathrm{LOD}$ \\
\hline (e,e)-2,4-hexadiene & $<$ LOD & $<\mathrm{LOD}$ & $<\mathrm{LOD}$ & $0.84^{\mathrm{b}}$ & $4.21^{\mathrm{a}}$ & $<\mathrm{LOD}$ & $0.85^{\mathrm{b}}$ & $<\mathrm{LOD}$ & $<\mathrm{LOD}$ \\
\hline \multicolumn{10}{|c|}{ Terpenes } \\
\hline Alpha-pinene & $38.47^{\mathrm{ab}}$ & $5.72^{\mathrm{c}}$ & $64.78^{\mathrm{a}}$ & $5.11^{\mathrm{c}}$ & $4.10^{\mathrm{c}}$ & $9.20^{\mathrm{bc}}$ & $14.00^{\mathrm{bc}}$ & $0.89^{\mathrm{c}}$ & $6.61^{\mathrm{c}}$ \\
\hline Alpha-thujene & $5.28^{\mathrm{a}}$ & $<$ LOD & $<\mathrm{LOD}$ & $<\mathrm{LOD}$ & $<\mathrm{LOD}$ & $2.99^{\mathrm{a}}$ & $<\mathrm{LOD}$ & $<\mathrm{LOD}$ & $<\mathrm{LOD}$ \\
\hline Camphene & $4.99^{\mathrm{a}}$ & $4.98^{\mathrm{a}}$ & $0.72^{\mathrm{b}}$ & $0.11^{\mathrm{b}}$ & $<\mathrm{LOD}$ & $0.83^{\mathrm{b}}$ & $<\mathrm{LOD}$ & $<\mathrm{LOD}$ & $0.25^{\mathrm{b}}$ \\
\hline Beta-pinene & 67.54 & 16.46 & 17.48 & 15.40 & 8.04 & 30.14 & 3.50 & $<\mathrm{LOD}$ & 21.94 \\
\hline Sabinene & 11.78 & 6.86 & 3.19 & 3.12 & 3.82 & 8.05 & 0.85 & 0.16 & 4.28 \\
\hline Myrcene & 9.06 & 6.46 & 8.81 & 8.20 & 4.81 & 6.97 & 1.29 & $<\mathrm{LOD}$ & 6.30 \\
\hline Limonene & 274.20 & 214.68 & 151.72 & 231.92 & 194.44 & 278.84 & 36.61 & 2.59 & 296.07 \\
\hline Alpha-phellandrene & 0.87 & 5.44 & $<\mathrm{LOD}$ & $<\mathrm{LOD}$ & $<\mathrm{LOD}$ & 3.89 & $<\mathrm{LOD}$ & $<\mathrm{LOD}$ & $<\mathrm{LOD}$ \\
\hline Beta-pinene & 2.33 & $<\mathrm{LOD}$ & $<\mathrm{LOD}$ & $<$ LOD & $<\mathrm{LOD}$ & $<\mathrm{LOD}$ & $<\mathrm{LOD}$ & 0.40 & $<\mathrm{LOD}$ \\
\hline Alpha-terpinolene & $5.96^{\mathrm{ab}}$ & $5.18^{\mathrm{ab}}$ & $3.09^{\mathrm{ab}}$ & $1.84^{\mathrm{b}}$ & $0.97^{\mathrm{b}}$ & $9.01^{\mathrm{a}}$ & $0.79^{\mathrm{b}}$ & $0.18^{\mathrm{b}}$ & $4.45^{\mathrm{ab}}$ \\
\hline Alpha-terpinene & 1.91 & 3.52 & 1.15 & 1.19 & 0.90 & 3.13 & $<\mathrm{LOD}$ & $<\mathrm{LOD}$ & 1.92 \\
\hline Gamma-terpinene & $86.27^{\mathrm{a}}$ & $61.91^{\mathrm{ab}}$ & $4.94^{\mathrm{b}}$ & $<\mathrm{LOD}$ & $13.31^{\mathrm{ab}}$ & $87.20^{\mathrm{a}}$ & $1.93^{\mathrm{b}}$ & $0.25^{\mathrm{b}}$ & $41.66^{\mathrm{ab}}$ \\
\hline Linalool & $161.12^{\mathrm{a}}$ & $109.85^{\mathrm{ab}}$ & $94.57^{\mathrm{abc}}$ & $86.93^{\mathrm{abc}}$ & $69.62^{\mathrm{bcd}}$ & $137.30^{\mathrm{ab}}$ & $12.81^{\mathrm{cd}}$ & $<\mathrm{LOD}$ & $<\mathrm{LOD}$ \\
\hline Beta-terpineol & $2.21^{\mathrm{c}}$ & $1.07^{\mathrm{d}}$ & $5.51^{\mathrm{a}}$ & $<\mathrm{LOD}$ & $<\mathrm{LOD}$ & $2.85^{\mathrm{b}}$ & $<\mathrm{LOD}$ & $<\mathrm{LOD}$ & $<\mathrm{LOD}$ \\
\hline Eugenol & $153.56^{\mathrm{a}}$ & $52.30^{\mathrm{bc}}$ & $28.00^{\mathrm{cd}}$ & $35.64^{\mathrm{c}}$ & $29.94^{\text {cd }}$ & $77.11^{\mathrm{b}}$ & $<\mathrm{LOD}$ & $<\mathrm{LOD}$ & $1.37^{\mathrm{d}}$ \\
\hline Terpinen-4-ol & $63.45^{\mathrm{a}}$ & $7.43^{\mathrm{b}}$ & $1.40^{\mathrm{b}}$ & $<\mathrm{LOD}$ & $<\mathrm{LOD}$ & $6.88^{\mathrm{b}}$ & $<\mathrm{LOD}$ & $<\mathrm{LOD}$ & $<\mathrm{LOD}$ \\
\hline Trans-isoeugenol & $<\mathrm{LOD}$ & $<\mathrm{LOD}$ & $<\mathrm{LOD}$ & $1.85^{\mathrm{b}}$ & $1.33^{\mathrm{c}}$ & $3.51^{\mathrm{a}}$ & $<\mathrm{LOD}$ & $<\mathrm{LOD}$ & $<\mathrm{LOD}$ \\
\hline Eucalyptol & $<\mathrm{LOD}$ & $9.24^{\mathrm{ab}}$ & $2.24^{\mathrm{b}}$ & $<\mathrm{LOD}$ & $<\mathrm{LOD}$ & $12.01^{\mathrm{a}}$ & $<\mathrm{LOD}$ & $<\mathrm{LOD}$ & $<\mathrm{LOD}$ \\
\hline Alpha-terpineol & $25.62^{\mathrm{b}}$ & $3.36^{\mathrm{b}}$ & $12.35^{\mathrm{b}}$ & $7.42^{\mathrm{b}}$ & $6.10^{\mathrm{b}}$ & $189.03^{\mathrm{a}}$ & $0.99^{\mathrm{b}}$ & $<\mathrm{LOD}$ & $<\mathrm{LOD}$ \\
\hline
\end{tabular}

${ }^{\text {abcd }}$ Values reported are the mean of two replicates. Mean values for each volatile compound in the same row followed by different letters differ significantly ( $p$-value < 0.05). LOD: Above the detection limit.

Of the total volatile compounds detected, benzaldehyde and hexanal discriminated between UHT samples and pasteurized. The latter did not present these compounds. Our data corroborated with the work of Potineni and Peterson [17] which demonstrated that the thermal treatment level in the samples had a definite effect on the stability of benzaldehyde. These authors found more than $90 \%$ of the benzaldehyde oxidized in milk pasteurized samples, in contrast to UHT milk samples, where no degradation of benzaldehyde was observed over 6 days [17].

According to literature, low levels of aldehydes and ketones are found in pasteurized sample, which shows that most of these compounds are formed during heat treatment. For that reason, their increase in UHT samples could be related to the increase in the severity of heat treatment [18]. In contrast, the highest level of 2-butanone was observed in pasteurized sample. In this case, similarly with 1-octanol, which was only detected in the pasteurized sample, their formation could be related to the metabolism of microbial activity [19], suggesting that the lower heat treatment made with pasteurization could provide only a partial destruction of the microorganisms responsible for their formation.

Terpenes were the predominant functional group in all samples, whereas alpha-pinene, beta-pinene, alphaterpinolene and limonene were found in all of them. Limonene compound presented the most intense response of the entire volatile profile drawn in each of the samples analyzed, except for OXT8. It is difficult to comment on whether the relative level of this compound is in agreement with other studies since this is the first time that the volatile profile of tiger nut beverage has been described.

The compound 6-methyl-5-hepten-2-one was the ketone with the highest response, and it has been characte- 
rized as having a fruit-like aroma [20].

Another functional group which contributes to the profile of volatile tiger nut beverage is alcohols, being ethanol and 1-nonanol those which presented the highest response in UHT.

Furans are volatile heterocyclic compounds which are formed during the heat treatment of foods and drinks as one of the Maillard reaction products [21]. From the obtained results it can be noticed that significant amounts of furans were generated during the thermal decomposition of sugars, known as caramelization, a non-enzymatic carbohydrate browning reaction, which commonly occurs during food processing [21]. In a study by OwczarekFendor et al. [22], furan formation from carbohydrates has been mostly studied in the presence of amino acids or whey proteins. The presence of proteins significantly increase the amounts of furans formed in starch-based model systems containing carbohydrates in combination with whey proteins. According to our results this behavior was not observed, since the samples OXT8 and OXT9 were those that had no milk proteins added to their composition compared to the other samples. However, the literature demonstrates multiple sources of furan formation being the primary source of the thermal degradation of carbohydrates such as fructose, glucose and lactose [21], or even starch [23]. The latest author noted that volatiles such as pyrazines, furans, aromatic compounds, pyrrole and thiazole were produced from Strecker degradation in Maillard reactions and gave rise to a nutty, roasted or bready aroma.

Nineteen compounds were found in the group of aromatic compounds. Among them, isomers of dimethylethylbenzene and trimethylbenzenes were detected. Trimethylbenzenes, ethylbenzene and toluene could be derived from the thermal degradation of sugar or amino acid [24].

\section{Conclusion}

Our results show that the method based on HS-SPME/GC-MS optimized for this work seems to be very useful for characterizing the volatile profile of "tiger nut beverage" (orxata de xufla). The optimization method established that the fiber DVB/CAR/PDMS presented the most efficient extraction of compounds from the headspace. An extraction temperature of $60^{\circ} \mathrm{C}$ and $2 \mathrm{~mL}$ of the volume sample assured the best transfer of volatiles. The addition of salt did not show a significant advantage for the qualification of the volatile fraction.

\section{References}

[1] Ferragut, V., Hernández-Herrero, M., Poliseli, F., Valencia, D. and Guamis, B. (2011) Ultra High Pressure Homogenization (UHPH) Treatment of Vegetable Milks: Improving Hygienic and Colloidal Stability. Proceedings of the 11th International Congress on Engineering and Food, Athens, 22-26 May 2011, 40-41.

[2] (2013) Ministerio de Agricultura, Alimentación y Mediambiente. Superfícies y producciones de cultivo. http://www.magrama.gob.es/estadistica/pags/anuario/2011/AE_2011_13_03_03_03.pdf

[3] (2013) CRDO Consejo Regulador de la Denominación de Origen Chufa de Valencia. http://www.chufadevalencia.org

[4] Corrales, M., Souza, P.M., Stahl, M.R. and Fernández, A. (2012) Effects of the Decontamination of a Fresh Tiger Nut Milk Beverage (Horchata) with Short Wave Ultraviolet Treatments (UV-C) on Quality Attributes. Innovative Food Science \& Emerging Technologies, 13, 163-168. http://dx.doi.org/10.1016/j.ifset.2011.07.015

[5] Cortés, C., Esteve, J.M., Frigola, A. and Torregros, F. (2005) Quality Characteristics of Horchata (a Spanish Vegetable Beverage) Treated with Pulsed Electric Fields during Shelf-Life. Food Chemistry, 91, 319-325. http://dx.doi.org/10.1016/j.foodchem.2004.06.014

[6] Perez-Cacho, P.R. and Rouseff, R. (2008) Processing and Storage Effects on Orange Juice Aroma. A Review. Journal of Agricultural and Food Chemistry, 56, 9785-9796. http://dx.doi.org/10.1021/jf801244j

[7] Bazemore, R., Goodner, K.L. and Rouseff, R.L. (1999) Volatiles from Unpasteurized and Excessively Heated Orange Juice Analyzed with Solid Phase Microextraction and GC-Olfactometry. Journal of Food Science, 64, 800-803. http://dx.doi.org/10.1111/j.1365-2621.1999.tb15915.x

[8] Ducki, S., Miralles-Garcia, J. and Zumbe, A. (2008) Evaluation of Solid-Phase Micro-Extraction Coupled to Gas Chromatography-Mass Spectometry for the Headspace Analysis of Volatile Compounds in Cocoa Products. Talanta, 74, 1166-1174. http://dx.doi.org/10.1016/j.talanta.2007.08.034

[9] Kataoka, H., Lord, H.L. and Pawliszyn, J. (2002) Applications of Solid-Phase Microextraction in Food Analysis. Journal of Chromatography A, 880, 35-62. http://dx.doi.org/10.1016/S0021-9673(00)00309-5

[10] McLafferty, F.W. (2000) Wiley Registry of Mass Spectral Data. 6th Edition.

[11] R Development Core Team (2005) R Foundation for Statistical Computing. Vienna. 
[12] Geerdink, R.B., Breidenbach, R. and Epema, O.J. (2007) Optimization of Headspace Solid-Phase Microextraction Gas Chromatography-Atomic Emission Detection Analysis of Monomethylmercury. Journal of Chromatography A, 1174, 7-12. http://dx.doi.org/10.1016/j.chroma.2007.08.070

[13] Nongonierma, A., Cayot, P., Le Quéré, J.L., Springett, M. and Voilley, A. (2006) Mechanisms of Extraction of Aroma Compounds from Foods, Using Adsorbents: Effect of Various Parameters. Food Reviews International, 22, 51-94. http://dx.doi.org/10.1080/87559120500379951

[14] Machields, D. and Istasse, L. (2003) Evaluation of Two Commercial Solid-Phase Microextraction Fibers for the Analysis of Target Aroma Compounds in Cooked Beef Meat. Talanta, 61, 529-537. http://dx.doi.org/10.1016/S0039-9140(03)00319-9

[15] Prosen, H. and Zupancic-Kralj, L. (2002) Solid Phase Microextraction. Trends in Analytical Chemistry, 18, $272-282$. http://dx.doi.org/10.1016/S0165-9936(98)00109-5

[16] Guichard, E. (2000) Interactions between Flavor Compounds and Food Ingredients and Their Influence on Flavor Perception. Food Reviews International, 18, 49-70. http://dx.doi.org/10.1081/FRI-120003417

[17] Potineni, R.V. and Peterson, D.G. (2005) Influence of Thermal Processing Conditions on Flavor Stability in Fluid Milk: Benzaldehyde. Journal of Dairy Science, 88, 1-6. http://dx.doi.org/10.3168/jds.S0022-0302(05)72655-2

[18] Fernandez, X., Kerverdo, S., Duñach, E. and Lizzani-Cuvelier, L. (2002) Les hétérocycles dans la chimie des arômes. In: Société Française de Chimie, Ed., L'Actualité Chimique, Paris.

[19] Serhan, M., Linder, M., Hosri, C. and Fanni, J. (2010) Changes in Proteolysis and Volatile Fraction during Ripening of Darfiyeh, a Lebanese Artisanal Raw Goat’s Milk Cheese. Small Ruminant Research, 90, 75-82. http://dx.doi.org/10.1016/j.smallrumres.2010.01.008

[20] Kazeniac, S.J. and Hall, R.M. (1970) Flavor Chemistry of Tomato Volatiles. Journal of Food Science, 35, 519-530. http://dx.doi.org/10.1111/j.1365-2621.1970.tb04799.x

[21] Maga, J.A. and Katz, I. (1979) Furans in Foods. Critical Reviews in Food Science and Nutrition, 11, 355-400. http://dx.doi.org/10.1080/10408397909527268

[22] Owczarek-Fendor, A., Meulenaer, B., Scholl, G., Adams, A., Van Lancker, F., Eppe, G., et al. (2010) Furan Formation in Starch-Based Model Systems Containing Carbohydrates in Combination with Proteins, Ascorbic Acid and Lipids. Food Chemistry, 133, 816-821. http://dx.doi.org/10.1016/j.foodchem.2012.01.098

[23] Hodge, J.E., Mills, J.D. and Fischer, B.E. (1972) Compounds from Browned Flavour Derived from Sugar-Amine Reactions. Cereal Science Today, 17, 34-38.

[24] Keyhani, A. and Yalayan, V.A. (1996) Pyrolysis/GC/MS Analysis of N-(1-Deoxy-D-Fructos-1-yl)-L-Phenylalanine: Identification of Novel Pyridine and Naphthalene Derivatives. Journal of Agricultural and Food Chemistry, 44, 223229. http://dx.doi.org/10.1021/jf950418u

[25] Wu, Y., Zhu, B., Tu, C., Duan, C.Q. and Pan, Q.H. (2011) Generation of Volatile Compounds in Litchi Wine during Winemaking and Short-Term Bottle Storage. Journal of Agricultural and Food Chemistry, 59, 4923-4931. http://dx.doi.org/10.1021/jf2001876

[26] Ren, B. (2003) Atom-Type-Based AI Topological Descriptors for Quantitative Structure Retention Index Correlations of Aldehydes and Ketones. Chemometrics and Intelligent Laboratory Systems, 66, 29-39. http://dx.doi.org/10.1016/S0169-7439(03)00004-2

[27] Liu, C., Cheng, Y., Zhang, Y.H., Deng, X., Chen, F. and Xu, J. (2012) Volatile Constituents of Wild Citrus Mangshanyegan (Citrus nobilis Lauriro) Peel Oil. Journal of Agricultural and Food Chemistry, 60, 2617-2628. http://dx.doi.org/10.1021/jf2039197

[28] Larrayoz, P., Addis, M., Gauch, R. and Bosset, J.O. (2011) Comparison of Dynamic Headspace and Simultaneous Distillation Extraction Techniques Used for the Analysis of the Volatile Components in Three European PDO Ewes' Milk Cheeses. International Dairy Journal, 11, 911-926. http://dx.doi.org/10.1016/S0958-6946(01)00144-3

[29] Hashizume, M., Gordon, M.H. and Mottram, D.S. (2007) Light-Induced Off-Flavor Development in Cloudy Apple Juice. Journal of Agricultural and Food Chemistry, 55, 9177-9182. http://dx.doi.org/10.1021/jf0715727

[30] Ong, P.K., Acree, T.E. and Lavin, E.H. (1998) Characterization of Volatiles in Rambutan Fruit (Nephelium lappaceum L.). Journal of Agricultural and Food Chemistry, 46, 611-615. http://dx.doi.org/10.1021/jf970665t

[31] Neffat, M., Sriti, J., Hamdaoui, G., Kchouk, M.E. and Marzouk, B. (2011) Salinity Impact on Fruit Yield, Essential Oil Composition and Antioxidant Activities of Coriandrum sativum Fruit Extracts. Food Chemistry, 124, 221-225. http://dx.doi.org/10.1016/j.foodchem.2010.06.022

[32] El-Sayed, A.M. (2012) The Pherobase: Database of Pheromones and Semiochemicals.

[33] Acree, T. and Arn, H. (2004) Flavornet and Human Odor Space. http://www.flavornet.org/flavornet.html 